\title{
Minaccia dell'autostima e fantasie grandiose nel narcisismo non patologico
}

Guido Veronese ${ }^{1}$, Giovanni M. Ruggiero², Sandra Sassaroli², Marco Castiglioni ${ }^{1}$,

Sommario: Lo studio esplora gli aspetti qualitativi di: a) memorie di minaccia dell'autostima; b) fantasie grandiose e c) scenari rilassanti indotti sperimentalmente in un campione non clinico. Un gruppo di 103 giovani studenti universitari ha fornito e trascritto memorie personali di minaccia all'autostima, fantasie grandiose e scene rilassanti. Una scala a 5 punti è stata costruita ad hoc per consentire a 3 giudici indipendenti di valutare i contenuto biologico, psicologico e relazionale di ciascun testo. L'accordo tra giudici era soddisfacente. Le memorie di minaccia all'autostima e le fantasie grandiose presentavano un contenuto soprattutto relazionale, mentre gli scenari rilassanti erano caratterizzati da elementi relazionali e biologici. Per quanto riguarda la dimensione biologica, è emersa una correlazione significativa tra memorie di minaccia e fantasie grandiose.

Parole chiave: Fattori socio-relazionali, Minaccia all'autostima, Fantasie grandiose, Narcisismo.

Abstract: The study explored the qualitative features of: a) memories of threat to self-esteem, b) grandiose fantasies and c) relaxing scenarios, experimentally induced in a non-clinical participant sample. A group of 103 young university students produced and transcribed personal memories of threat to self-esteem, grandiose fantasies and relaxing scenes. A five point scale was designed ad hoc to enable three independent raters to assess the biological, psychological and relational contents of each of the texts. Inter-rater agreement was satisfactory. Memories of threat to self-esteem and grandiose fantasies were mainly sociorelational, while relaxing scenarios were characterised by both relational and biological elements. With reference to the biological dimension, there was also significant correlation between memories of threat to self-esteem and grandiose fantasies.

1 Facoltà Scienze della Formazione, Università Milano Bicocca; 2 "Studi Cognitivi", scuola di specializzazione post-laurea in psicoterapia cognitiva Milano, Italia; "Psicoterapia Cognitiva e Ricerca", scuola di specializzazione post-laurea in psicoterapia cognitiva, Milano, Italia

Corrispondenza: Guido Veronese, Facoltà di Scienze della Formazione, Università Milano Bicocca, Piazza dell'Ateneo 1, 20126, Milano, Italia. E-mail: guido.veronese@unimib.it 


\section{Introduzione}

Secondo il DSM IV-TR (APA, 2000), tra i caratteri distintivi dei pazienti sofferenti di Disturbo Narcisistico di Personalità (DNP) vi sono fantasie grandiose di potere, successo e superiorità, il sentimento che tutto sia loro dovuto e la mancanza di apprezzamento verso i successi e qualità altrui (Dimaggio, Fiore, Salvatore e Carcione, 2007). Horowitz (1989) ha rilevato come lo stato di grandiosità sembri essere una manovra difensiva contro vissuti di bassa autostima e come tale stato maschererebbe un profondo senso di inferiorità e vergogna. Attraverso fantasie grandiose il narcisista tenterebbe disperatamente di evitare vissuti di critica e umiliazione per lui insopportabili (Bateman 1998; Bursten, 1989; Dimaggio et al., 2006; Kernberg, 1975; Kohut, 1971, 1977; Modell, 1984; Ryle e Kerr, 2002; Twenge e Campbell, 2003; Westen, 1990). I soggetti narcisisti si sentono profondamente esclusi, disprezzati e ostracizzati (Dimaggio et al., 2007; Dimaggio e Semerari, 2003; Dimaggio et al,. 2002).

Ruggiero, Tudisco e Sassaroli (2007) hanno fornito evidenze sperimentali circa la interconnessione di fantasie grandiose e fantasie di minaccia dell'autostima in un campione non clinico. L'esperimento consisteva nella richiesta di segnalare, alzando una mano, le intrusioni ideative di bassa autostima durante stati mentali autoindotti di grandiosità e neutralità rilassata. Gli stati grandiosi mostravano un numero di intrusioni significativamente più elevato dell'evocazione di scenari rilassanti. Questo dato appoggia l'ipotesi della connessione tra grandiosità narcisistica e dubbi di autostima.

In una recente rassegna della letteratura teorica e empirica viene discussa l'ipotesi di una autostima fragile nelle personalità narcisistiche (Bosson, Lakey, Campbell, Zeiger-Hill, Jordan e Kernis, 2008). Gli autori notano come, nonostante molti studi confermino come un'alta autostima manifesta mascheri un'autostima implicita bassa, altri studi non siano riusciti a replicare questo modello. Alcuni studi hanno rilevato una relazione inversa tra narcisismo e autostima (Rose, 2002; Soyer et al., 2002), mentre altri studi hanno riportato un'associazione positiva (Raskin, Terry, 1998; Raskin, Novacek e Hogan, 1991). Bosson e collaboratori propongono alcune ipotesi alternative per spiegare risultati altrimenti inconsistenti: a) un'ipotesi alternativa è quella che esistano due sottotipi di narcisismo, grandioso e vulnerabile; b) una seconda ipotesi è che il narcisista mostri una forte e solida autostima in compiti di performance (es. nello studio e al lavoro) e una fragile autostima nel dominio affettivo e relazionale (Crocker, Luhtanen e Cooper, 2003). Un'ulteriore spiegazione è che il narcisista possa mostrare autostima sia bassa che alta, lasciando immaginare che la sua autostima possa essere buona anche se facilmente minacciabile e fluttuante, in costante ricerca di validazione e fluttuante e mantenute attraverso strategie di auto-illusione (Kernis, 2003). Perciò è possibile che il narcisismo correli con un'autostima molto alta ma instabile (Kernis et al., 1993). Inoltre questa instabilità è associata a una reattività emozionale molto alta nell'affrontare eventi con implicazioni negative per il sé (Rhodewalt, Madrian e Cheney, 1998; Westen, 1990).

Riassumendo, le ricerche empiriche non danno una definitiva conferma dell'ipotesi di una bassa autostima mascherata nel narcisista, impedendo di 
tracciare una definitiva generalizzazione di questo modello. Tuttavia alcuni dati confermano come individui con alti livelli di narcisismo mostrino componenti di fragilità nella loro autostima, suggerendo la possibilità di estendere a un determinato sottogruppo di narcisisti l'ipotesi. Riteniamo possibile che a questo sottogruppo appartengano quegli individui che accedono alla psicoterapia.

A prescindere dalle differenti interpretazioni qui esposte, sembra esserci un ampio consenso rispetto al ruolo cruciale giocato dai domini interpersonale e sociale nel determinare le dinamiche di autostima del narcisista. Non si conosce molto rispetto alla qualità delle fantasie grandiose e delle cadute di autostima in individui con DPN. Seguendo quelle teorie che enfatizzano l'importanza delle relazioni sociali nel determinare l'origine, sviluppo e mantenimento del Sé e dell'identità personale, sia essa tipica o atipica (Bateson, 1972; FivazDepeursinge e Corboz-Warnery, 2000; Haley, 1971 ; Schaffer, 1989; Ugazio, 1998; Ugazio et al., 2008; Ugazio e Castiglioni, 1998), obiettivo di questo lavoro è investigare se la dimensione interpersonale giochi un ruolo preminente di altre dimensioni, e nello specifico della dimensione biologico-corporea e psicologicointrapsichica, nella costruzione di memorie di minaccia dell'auto-stima, di fantasie grandiose e di scenari rilassanti.

Le tre dimensioni considerate sono estratte a partire dal modello biopsico-sociale di Engel (1977, 1980), una teoria generale del funzionamento umano utile nel descrivere e comprendere l'esperienza soggettiva della malattia (Epstein e Borrell-Carrio, 2005). Il modello identifica dimensioni biologiche, psicologiche e sociali come categorie per classificare e esplorare la realtà esterna come gli stati interni (Gabbard, 2000, 2005; Engel, 1977; Epstein e BorrellCarrio, 2005).

La nostra idea di fondo è che l'individuo non sia un passivo spettatore di ciò che gli accade - internamente e esternamente - (realtà oggettiva), ma che esso conosca e esperisca attraverso processi mentali attivi e costruttivi (Toomey e Eker, 2009, 2007; Eker e Toomey, 2008). Percezioni del sé biologico, psicologico e relazionale sono la risultante di una personale attività interpretativa cerebrale, che consente all'individuo di anticipare eventi interni ed esterni grazie a attività di specifici campi recettivi neurali (Toomey e Eker, 2007). Il sistema cervellomente-corpo è ingaggiato in una continua attività costruttiva e ri-costruttiva informata dall' esperienza, per controllare, tollerandole, contraddizioni senza che esse disconfermino la struttura portante del sistema costruttivo personale (Eker e Toomey, 2008). Un approccio costruttivita ci consente di considerare la complessità del sé evitando dicotomie riduzioniste implicite nella polarizzazione del dibattito scientifico quali mente versus cervello, processi top-down versus processi bottom-up (Toomey e Eker, 2009).

Abbiamo utilizzato un modello tridimensionale bio-psico-sociale per effettuare una analisi qualitativa delle esperienze soggettive di: a) fantasie grandiose, b) memorie di minaccia dell'autostima, c) scenari rilassanti. Tutte e tre le scene erano indotte in un campione subclinico durante un esperimento descritto nella sezione Metodo.

Nell'ambito di tale cornice teorica, le nostre ipotesi erano:

1. La dimensione relazionale dovrebbe essere prominente in tutte e tre $\mathrm{i}$ tipi di scena (minaccia, grandiosa, rilassante). 
2. Le fantasie grandiose e le scene di minaccia dell'autostima dovrebbero avere un'analogia di struttura e risultare differenti dallo scenario rilassante. Nello specifico riteniamo che tale analogia si evidenzi attraverso una distribuzione quantitativa delle dimensioni biologica, psicologica e relazionale simile nella scena di minaccia e grandiosa, una distribuzione completamente diversa delle dimensioni biologica, psicologica e relazionale nella scena rilassante.

3. Infine si ipotizza che $\mathrm{i}$ soggetti tendano più frequentemente a connettere scene di minaccia e fantasie grandiose, mantenendo lo stesso contenuto narrativo nelle scene grandiose e di minaccia ( per es.: alla descrizione di un fallimento scolastico, segue la fantasia grandiosa di ricevere un premio scientifico internazionale che riscatti l'individuo dalla cocente delusione). Di contro ci aspettiamo che il contenuto narrativo delle scene rilassanti sia del tutto differente rispetto alle scene di minaccia e rilassanti.

\section{Metodo}

\subsection{Soggetti, Strumenti e procedura}

A 130 studenti universitari milanesi sono stati preliminarmente valutati con il colloquio clinico basato sui criteri del DSM-IV al fine di eliminare i soggetti con livelli patologici di disturbo psichico. Sono stati esclusi 27 soggetti con disturbo d'ansia e/o dell'umore. In un caso era presente un disturbo di adattamento. I 103 soggetti selezionati (età media 22 anni, range 21-27 anni; 94 femmine e 9 maschi) dovevano quindi, su indicazione di uno sperimentatore, rievocare mentalmente l'episodio della loro vita in cui avevano sentito maggiormente minacciata la loro autostima; dopo un minuto veniva loro chiesto di produrre una fantasia grandiosa e di concentrarsi intensamente su di essa per altrettanto tempo. Infine, trascorsi i successivi sessanta secondi, lo sperimentatore richiedeva di concentrarsi su una scena altamente rilassante. Si chiedeva a ogni soggetto di segnare su una griglia costruita ad hoc il numero di intrusioni della scena di minaccia dell'autostima percepite durante l'immaginazione della fantasia grandiosa e della scena rilassante. I soggetti infine dovevano descrivere dettagliatamente per iscritto su un apposito foglio il contenuto delle scene da essi immaginate.

Le descrizioni così ottenute sono state codificate, in accordo con il modello bio-psico-sociale (Engel, 1977, 1980; Epstein e Borrell-Carrio, 2005; Gabbard, 2000, 2005), da 3 giudici indipendenti (due psicologi sistemico-costruzionisti e uno psicologo cognitivista) in relazione a 3 macrodimensioni: 1) biologicocorporea; 2) psicologico-intrapsichica; 3) relazionale-sociale. Il grado di accordo intergiudici è risultato pari a 0.80 . Tale accordo è il risultato dell'accordo medio tra dimensioni macro e accordo medio delle sottodimensioni (vedi tabella 1) nelle scene di minaccia, grandiosa e di rilassamento. Per quanto riguarda le dimensioni biologico, psicologico e relazionale nelle tre scene l'accordo medio tra giudici era rispettivamente di $0,81,0,80$ e 0,78 . 
Ricerca in Psicoterapia / Research in Psychotherapy: Psychopathology, Process and Outcome 2009; 1-2 (12): www.spr-italia.it/pdf/spr_volume_12.pdf

Tabella 1: Accordo intergiudici di tutte le sottodimensioni nelle scene grandiosa, di minaccia e rilassante

\begin{tabular}{|c|c|c|c|}
\hline & $\begin{array}{c}\text { Scena } \\
\text { grandiosa }\end{array}$ & $\begin{array}{l}\text { Scena di } \\
\text { minaccia }\end{array}$ & $\begin{array}{c}\text { Scena } \\
\text { rilassante }\end{array}$ \\
\hline Descrizione corporea & 0,90 & 0,65 & 0,88 \\
\hline Sensazione corporea & 0,80 & 0,78 & 0,77 \\
\hline Arousal fisiologico/attivazione & 0,78 & 0,82 & 0,76 \\
\hline Auto-efficacia percepita & 0,72 & 0,90 & 0,80 \\
\hline Stabilità emotiva & 0,71 & 0,73 & 0,78 \\
\hline Sviluppo intellettualee cultura & 0,81 & 0,88 & 0,84 \\
\hline Internalizzazione & 0,70 & 0,65 & 0,81 \\
\hline Esternalizzazione & 0,86 & 0,60 & 0,80 \\
\hline $\begin{array}{l}\text { Relazioni professionali e del tempo } \\
\text { libero }\end{array}$ & 0,85 & 0,88 & 0,78 \\
\hline Relazioni sentimentali & 0,80 & 0,82 & 0,80 \\
\hline Relazioni Familiari & 0,80 & 0,85 & 0,77 \\
\hline Relazioni con altri significativi & 0,83 & 0,89 & 0,81 \\
\hline $\begin{array}{l}\text { Riconoscimento professionale e } \\
\text { successo }\end{array}$ & 0,82 & 0,78 & 0,81 \\
\hline Altre relazioni & 0,83 & 0,76 & 0,73 \\
\hline
\end{tabular}

Ciascuna macrodimensione constava di alcuni sottolivelli che ne specificano i differenti aspetti. La macrodimensione biologico-corporea si articolava in 3 sottolivelli: a) descrizioni del corpo; b) sensazioni corporee; c) attivazione fisiologica (es: "vedo il mio corpo grasso"; "sento il sole scaldarmi la pelle"; "il cuore mi batte all'impazzata").

All'interno della dimensione psicologica intrapsichica cadevano le scene in cui si rilevavano elementi descrittivi significativamente connessi a fattori interni all'individuo, quali aspetti cognitivi, emotivi, caratteristiche di personalità e di temperamento (es. "sono timido", "mi sento stupida", ecc.). 
Questa macrodimensione si articolava in 5 livelli, ispirandosi alla letteratura sui "Big Five" (Eysenck e Eysenck, 1985; Eysenck, 1991): a) autoefficacia percepita; b) emotività; c) intelligenza/cultura; d) internalizzazione; e) esternalizzazione.

All'interno della dimensione relazionale-sociale rientravano infine le scene in cui erano presenti persone in interazione significativa con il soggetto, ovvero tutte le situazioni sociali che contestualizzassero o determinassero l'immagine di sé del protagonista nella scena descritta (es. "le critiche di mia madre mi fanno sentire uno schifo"; "sono attorniato da persone che mi esaltano per la mia bellezza" ecc.). Erano 6 i sottolivelli in cui si articolava questa dimensione: a) relazioni professionali/ludiche/sportive; b) relazioni sentimentali; c) relazioni familiari; d) relazione con altri significativi (amici); e) Successo e autoaffermazione; f) altre relazioni. Veniva inoltre introdotta in questa macrodimensione un'ulteriore distinzione tra interazioni diadiche vs. interazioni triadiche/poliadiche. Si tratta di una variabile dicotomica per indicare se la scena descrivesse un'interazione a due (es. "la maestra mi umilia dicendomi che sono un asino"; "Il mio ragazzo non mi saluta perché ce l'ha con me") o più partecipanti ("la maestra mi umilia di fronte a tutta la classe"; "Il mio ragazzo non mi saluta perché è con un'altra"). Una quarta categoria, "altro", che si è rivelata statisticamente non rilevante, raccoglieva le descrizioni non ascrivibili alle tre precedentemente elencate. Le tre dimensioni di categorizzazione delle scene non erano mutualmente escludentesi. Esse potevano coesistere con un grado minore o maggiore di prevalenza, valutato su una scala Likert a 5 punti. Il punteggio zero indicava l'assenza della dimensione o sottodimensione biologica, psicologica e relazionale, il punteggio 5 (molto) indicava un netto emergere della dimensione biologica, psicologica e relazionale. Dalla descrizione delle scene potevano emergere più dimensioni e più sottodimensioni contemporaneamente.

\section{Risultati}

I dati ottenuti sono stati sottoposti ad ANOVA con analisi post hoc di Tukey (variabile indipendente: "tipo di scena"; variabile dipendente: "dimensioni" rilevate nelle descrizioni delle diverse scene), con i risultati qui sintetizzati.

La dimensione relazionale-sociale risulta statisticamente preminente rispetto alle altre due sia nella scena di minaccia sia nella scena grandiosa (vedi tab. 1 e figura 1); nella scena rilassante non si rilevano invece differenze statisticamente significative tra la dimensione relazionale-sociale e quella biologico-corporea. 
In figura 1 vengono rappresentati gli andamenti delle tre macrodimensioni nella scena di minaccia e nelle fantasie grandiose e rilassante.

Figura 1: Medie delle tre dimensioni, biologica, psicologica e relazionale, nelle diverse scene.

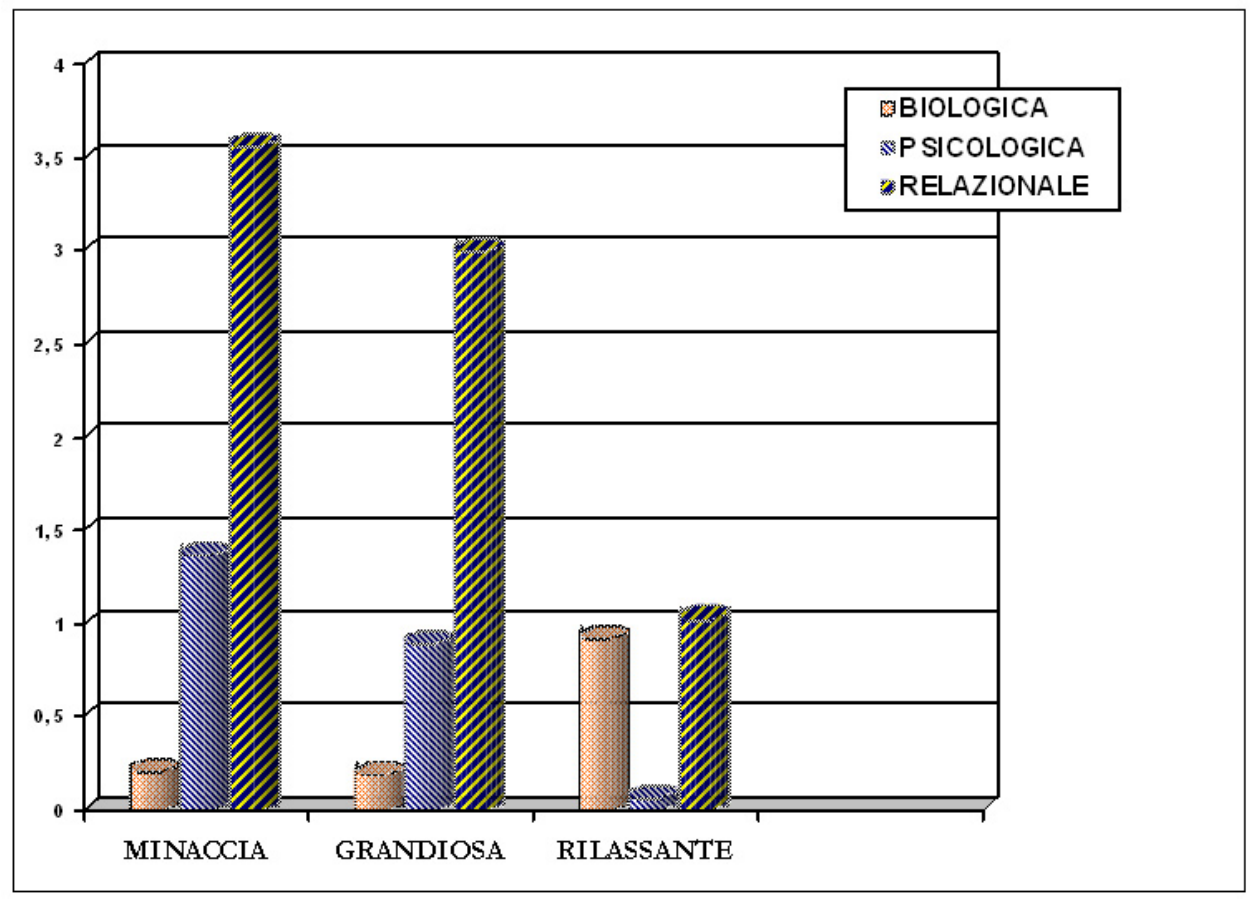

La tabella 2 illustra i livelli di significatività delle differenze tra le tre dimensioni (Biologico/Psicologico/Relazionale) nelle scena di minaccia e nelle fantasie grandiose e rilassante.

\begin{tabular}{lllll}
\multicolumn{6}{c}{ Tabella 2: ANOVA delle macrodimensioni nella scena di minaccia. } \\
\hline & N & \multicolumn{4}{l}{ Insiemi di punteggi * } \\
& & Psicologica & Biologica & Sociale \\
\hline Minaccia & 103 & $0,21^{\mathrm{a}}$ & $1,38^{\mathrm{b}}$ & $3,57^{\mathrm{c}}$ \\
Grandiosa & 103 & $0,19^{\mathrm{a}}$ & $0,87^{\mathrm{b}}$ & $2,99^{\mathrm{c}}$ \\
Rilassante & 103 & $0,08^{\mathrm{a}}$ & $0,92^{\mathrm{b}}$ & $1,03^{\mathrm{b}}$ \\
& & & & \\
\hline
\end{tabular}

Punteggi significativamente differenti hanno $p<0,05$

La descrizione di interazioni "triadiche/poliadiche" risulta minoritaria rispetto alla descrizione di interazioni diadiche. Si evidenzia però una differenza tra scene di minaccia (dove le interazioni tradico-poliadiche rasentano il 25\%), scene grandiose (scene triadico-poliadiche pari all'8\%) e scene rilassanti (scene tradico-poliadiche assenti).

Per studiare le eventuali analogie strutturali tra i diversi tipi di scene, è stata inoltre effettuata, attraverso l'indice $r$ di Pearson un'analisi delle correlazioni tra le dimensioni e le scene confrontate a due a due, che consentiva di rilevare l'andamento delle tre macrodimensioni rispetto ai tre tipi di scena 
L'andamento delle singole macrodimensioni nelle scene di minaccia e nelle fantasie di grandiosità risulta analogo, mentre differisce dall'andamento nella scena rilassante. In altre parole, esiste una sistematica correlazione tra le variazioni riscontrate nella scena di minaccia e nella fantasia grandiosa, mentre la scena rilassante risulta a se stante. La figura 1 mostra l'evidente analogia strutturale della scena di minaccia e della fantasia grandiosa.

Le seguenti tabelle (Tabelle 3-5) mostrano correlazioni delle scene rispetto alle tre dimensioni (corporea, psicologica e relazionale). La significatività delle covariazioni tra fantasie grandiose e scene di minaccia, fa pensare ad un'analogia di struttura tra le due scene. Tale significatività non è presente nelle correlazioni delle prime due con la scena rilassante che perciò appare "distaccata" dalle altre.

Tabella 3: Correlazione tra le dimensioni biologica, psicologica e relazionale nelle scene di minaccia e fantasie grandiose

\begin{tabular}{llll}
\hline & $\begin{array}{l}\text { Minaccia } \\
\text { Biologic } \\
\text { a }\end{array}$ & $\begin{array}{l}\text { Minaccia } \\
\text { Psicologi } \\
\text { ca }\end{array}$ & $\begin{array}{l}\text { Minaccia } \\
\text { Sociale }\end{array}$ \\
\hline Grandiosa Biologica & $0,733^{* *}$ & $-0,114$ & $-0,209^{*}$ \\
Grandiosa Psicologica & 0,006 & 0,073 & $-0,088$ \\
Grandiosa Sociale & 0,045 & 0,015 & $0,237^{*}$ \\
\hline$* * \mathrm{p}<0,01 ;{ }^{*} \mathrm{p}<0.05$ & & &
\end{tabular}

Tabella 4: Correlazione tra le dimensioni biologica, psicologica e relazionale nelle scene di minaccia e scena rilassante

\begin{tabular}{|c|c|c|c|}
\hline & $\begin{array}{l}\text { Minaccia } \\
\text { Biologic } \\
\text { a }\end{array}$ & $\begin{array}{l}\text { Minaccia } \\
\text { Psicologi } \\
\text { ca }\end{array}$ & $\begin{array}{l}\text { Minaccia } \\
\text { Sociale }\end{array}$ \\
\hline Rilassante Biologica & $-0,155$ & 0,092 & $0,004 * *$ \\
\hline Rilassante Psicologica & $-0,046$ & 0,088 & 0,064 \\
\hline Rilassante Sociale & 0,025 & $-0,014$ & $-0,71$ \\
\hline
\end{tabular}

Un terzo dei soggetti ha stabilito, a livello di contenuti descritti, connessioni tra scena di minaccia e scena grandiosa (34\%), mentre la scena rilassante risulta pressoché "isolata" (un solo soggetto stabiliva la connessione). Anche questo dato rivela una maggiore facilità nei soggetti a interrelare le fantasie grandiose e le scene di minaccia e la tendenza ad interrompere la continuità narrativa del processo di immaginazione durante il rilassamento.

Le stesse analisi di correlazione sono state effettuate sul solo sottocampione di soggetti che connettevano spontaneamente le scene. Sono stati rilevati risultati sovrapponibili a quelli forniti dall'intero gruppo di soggetti, seppure con un grado maggiore di significatività .

Tabella 5: Correlazione tra le dimensioni biologica, psicologica e relazionale nelle fantasie grandiose e scena rilassante

\begin{tabular}{llll}
\hline & \multicolumn{1}{c}{$\begin{array}{c}\text { Minaccia } \\
\text { Biologica }\end{array}$} & $\begin{array}{c}\text { Minaccia } \\
\text { Psicologica }\end{array}$ & $\begin{array}{c}\text { Minaccia } \\
\text { Sociale }\end{array}$ \\
\hline Rilassante Biologica & $-0,164$ & 0,028 & $-0,054$ \\
Rilassante Psicologica & $-0,042$ & 0,186 & $-0,049$ \\
Rilassante Sociale & 0,055 & 0,021 & 0,087 \\
\hline \multicolumn{1}{c}{$*{ }^{*} \mathrm{p}<0,01 ;{ }^{*} \mathrm{p}<0.05$} & & &
\end{tabular}


Infine, come ultima verifica dell'analogia strutturale tra scene di minaccia e fantasie grandiose e distanziamento delle scene rilassanti dalle prime due, sono state estratte e messe a confronto le sottodimensioni più pregnanti dei livelli corporeo, psicologico e relazionale per ciascuna scena. Le prime due descrizioni, di minaccia e grandiosa, mostrano perfetta sovrapponibilità anche per quanto riguarda le sottodimensioni salienti. La scena rilassante mostra, invece, una salienza dei sottolivelli del tutto autonoma. Se infatti per quanto riguarda il livello biologico la sottodimensione "Descrizione corporea" prevale nel ricordo minacciante l'autostima $(M=0,28 ; d s=0,98)$ e nella scena grandiosa $(M=0,17$; $\mathrm{ds}=0,8)$, nella scena rilassante prevale la "Sensazione corporea" $(M=0,96 ; \mathrm{ds}$ $=1,61)$. La sottodimensione prevalente del livello psicologico è l' "autoefficacia percepita" sia nella memoria di minaccia $(M=1,24 ; \mathrm{ds}=1,74)$ sia nella scena di grandiosità $(\mathbf{M}=0,56 ; \mathrm{ds}=1,33)$, sia nella scena rilassante $(\mathbf{M}=0,11 ; \mathrm{ds}=$ $0,62)$. Infine, la sottodimensione socio-relazionale prevalente nelle memorie minaccianti l'autostima $(\mathrm{M}=1,44 ; \mathrm{ds}=1,93)$ e nelle fantasie grandiose $(\mathrm{M}=$ $1,55 ; \mathrm{ds}=1,96)$ è "Relazioni professionali, ludiche e sportive", mentre nella scena rilassante $(\mathrm{M}=0,18 ; \mathrm{ds}=0,82)$ è la relazione con "Altri significativi".

\section{Conclusioni}

I risultati sembrano confermare l'ipotesi circa la pregnanza della dimensione relazionale-sociale, in particolare per quanto concerne l'immaginazione di scene di minaccia e le fantasie di grandiosità. Tale pregnanza viene conservata anche nella fantasia rilassante, ma viene in questo caso affiancata e pareggiata da contenuti di tipo biologico e corporeo che appaiono altrettanto salienti.

A livello clinico-interpretativo sembrerebbe possibile inferire come il "pubblico" (sia esso costituito da una o più persone) rivesta un ruolo fondamentale, tanto nelle situazioni di minaccia dell'autostima quanto in quelle di grandiosità (Cancrini, 2006; Dimaggio e Semerari, 2003; Dimaggio et al. 2002 ). I soggetti per rilassarsi si concentrano invece su fantasie che coinvolgono, oltre a quella relazionale, la dimensione fisica e corporea.

Per quanto concerne i dati riguardanti le interazioni triadiche/poliadiche, $i$ risultati confermano come i contesti triadici siano sostanzialmente estranei al "senso comune" (Ugazio, 1998; Ugazio et al., 2008): le persone pensano alle interazioni prevalentemente in termini diadici. Interessante però notare che la frequenza più alta di interazioni tradiche/poliadiche si registra sulla scena di minaccia dell'autostima.

Le analogie strutturali tra fantasie grandiose e scene di minaccia dell'autostima sono confermate (Horowitz, 1989). Le scene rilassanti rivelano, invece, una distribuzione delle dimensioni diversa. Ciò potrebbe fare presupporre che il tentativo del narcisista di proteggersi dalle conseguenze della minaccia alla propria autostima con manovre di tipo grandioso, non farebbe che rafforzare il senso di minaccia e il sentimento di inadeguatezza da cui cerca di proteggersi a ogni costo. Una strategia di distanziamento potrebbe essere invece individuata nel progressivo addestramento all' utilizzo di scenari di rilassamento. Molto probabilmente il narcisista si "rifugia" nella fantasia di 
grandiosità proprio perché fatica a proteggersi dall'incombente minaccia alla propria autostima attraverso strategie immaginative che lo aiutino a rilassarsi

L'intento della nostra ricerca esplorativa non era quello di individuare qualità e analogie strutturali di fantasie narcisistiche patologiche, ma di considerare le qualità di fantasie narcisistiche non patologiche e di rilassamento indotte in un campione di individui con personalità tipica. Qualità analoghe $\mathrm{e}$ funzionamenti diversi dovrebbero essere verificati in soggetti con DPN. Dunque, i risultati emersi, sebbene possano risultare utili per formulare ipotesi applicabili anche ad una popolazione di pazienti, non vanno generalizzati in quanto provenienti da un campione non clinico e nemmeno possono essere considerati definitivi sia per la distribuzione di genere del campione sia per la giovane età del gruppo di soggetti presi in esame.

\section{Bibliografia}

American Psychiatric Association. (2000). Diagnostic and statistical manual of mental disorders (4th ed., text rev. ed.) Washington DC: Author.

Bateman, A. W. (1998). Thick- and thin-skinned organisations and enactment in borderline and narcissistic disorders. International Journal of Psychoanalysis, 79, 13-22.

Bateson, G. (1972). Steps to an Ecology of Mind, London: Chandler Publishing Company.

Bosson, J. K., Lakey, C. E., Campbell, W. K. Zeigler-Hill, V., Jordan, C.H., \& Kernis, M.H. (2008). Untangling the Links between Narcissism and Self esteem: A Theoretical and Empirical Review. Social and Personality Psychology Compass, Disponibile online: http://www.blackwellsynergy.com/doi/abs/10.1111/j.1751-9004.2008.00089.x

Bursten, B. (1989). The relationship between narcissistic and antisocial personalities. In O. F. Kernberg (Ed.), Narcissistic personality disorder: Psychiatric Clinics of North America (pp. 571-584). Philadelphia: Saunders.

Cancrini, L. (2006). L'Oceano Borderline, Racconti di Viaggio, Milano: Raffaello Cortina.

Crocker, J., Luhtanen, R. K., Cooper, M. L., \& Bouvrette, A. (2003). Contingencies of self-worth in college students: Theory and measurement. Journal of Personality and Social Psychology, 85, 894-908.

Dimaggio, G., \& Semerari A. (a cura di) (2003). I Disturbi di Personalità. Modelli e Trattamento. Bari: Laterza.

Dimaggio, G., Fiore, D., Lysaker, P.H., Petrilli, D., Salvatore, G., Semerari, A., \& Nicolò, G. (2006) Early narcissistic transference patterns: an exploratory single case study from the perspective of dialogical self theory. Psychology and Psychotherapy, 79, 495-516.

Dimaggio, G., Fiore, D., Salvatore, G., \& Carcione, A. (2007). Dialogical relationship Patterns in Narcissistic Personalities: Session Analysis and Treatment Implications, Journal of Constructivist Psychology, 20, 1, pp. 23-51.

Dimaggio, G., Semerari, A., Falcone, M., Nicolò, G., Carcione, A., \& Procacci, M. (2002). Metacognition, states of mind, cognitive biases and interpersonal cycles. Proposal for an integrated model of Narcissism. Journal of Psychotherapy Integration, 4, 421-451. 
Ricerca in Psicoterapia / Research in Psychotherapy: Psychopathology, Process and Outcome 2009; 1-2 (12): www.spr-italia.it/pdf/spr_volume_12.pdf

Ecker, B., \& Toomey, B. (2008). Depotenziation of Symptom-Producing implicit memory in coherence therapy. Journal of Constructivist Psychology, 21, 2, 87-150.

Engel, G.L. (1977). The need for a new medical model: a challenge for biomedicine. Science, 196, 129-136.

Engel, G.L. (1980). The clinical application of the biopsychosocial model, American Journal of Psychiatry, 137, 535-544.

Epstein, R. M., \& Borrell-Carrio, F. (2005). The Biopsychosocial Model: Exploring Six Impossible Things. Families, Systems, \& Health, 23, 426-431.

Eysenck, H. J. (1991). Dimensions of personality: 16, 5, or 3? Criteria for a taxonomic paradigm. Personality and Individual Differences, 12, 773-790.

Eysenck, H. J., \& Eysenck, M. W.(1985). Personality and Individual Differences: a Natural Sciences Approach. New York: Plenum press.

Fivaz-Depeursinge, E., \& Corboz-Warnery (2000). IlTtriangolo Primario. Le Prime Interazioni Triadiche tra Padre, Madre e Bambino. Milano: Cortina.

Gabbard, G. (2000). A neurobiologically informed perspective on psychotherapy, British Journal of Psychiatry, 177, 117-122.

Gabbard, G. (2005). Mind, Brain and Personality Disorders, American Journal of Psychiatry, 162, 648-655.

Haley, J. (1971). Changing Families: a Family Therapy Reader. New York: Grune \& Stretton.

Horowitz, M. J. (1989). Clinical phenomenology of narcissistic Pathology. Psychiatric Clinics of North America, 12, 531-539.

Kernberg, O. F. (1975). Borderline Conditions and Pathological Narcissism. New York: American Psychiatric Press.

Kernis, M. H. (2003). Toward a conceptualization of optimal self-esteem. Psychological Inquiry, 14, 1-26.

Kernis, M. H., Cornell, D. P., Sun, C. R., Berry, A. J., \& Harlow, T. (1993). There's more to self-esteem than whether it is high or low: The importance of stability of self-esteem. Journal of Personality and Social Psychology, 65, 1190-1204.

Kohut, H. (1971). The Analysis of the Self. New York: International University Press.

Kohut, H. (1977). The Restoration of the Self. New York: International University Press.

Modell, A. H. (1984). Psychoanalysis in a New Context. New York: International University Press.

Raskin, R., \& Terry, H. (1988). A Principal-Components Analysis of the Narcissistic Personality Inventory and Further Evidence of Its Construct Validity. Journal of Personality and Social Psychology, 54, 890-902.

Raskin, R., Novacek, J., \& Hogan, R. (1991). Narcissistic self-esteem management. Journal of Personality and Social Psychology, 60, 911-918.

Rhodewalt, F., Madrian, J. C., \& Cheney, S. (1998). Narcissism, selfknowledge organization, and emotional reactivity: The effect of daily experiences on self-esteem and affect. Personality and Social Psychology Bulletin, $24,75-87$. 
Rose, P. (2002). The happy and unhappy faces of narcissism. Personality and Individual Differences, 33, 379 - 391.

Ruggiero, G.M., Tudisco A., \& Sassaroli S. (2007). Grandiose narcissistic daydreams stimulate low self-esteem worries: an experimental study. Paper presented at the SPR International, 38 ${ }^{\text {th }}$ International Meeting, Madison, WI, USA.

Ryle, A., \& Kerr, I. (2002). Introducing Cognitive Analytic Therapy. Principle and Practice. Chichester: Wiley.

Schaffer, H. R. (1984). Il Bambino e i suoi Partner: Interazione e Socialità. Ed. it. Milano: Angeli, (1994).

Soyer, R. B., Rovenpor, J. L., Kopelman, R. E., Mullins, L. S., \& Watson, P. J. (2001). Further assessment of the construct validity of four measures $f$ the narcissism: Replication and extension. The Journal of Psychology, 135, 245 258.

Toomey, B., \& Ecker, B. (2007). Of Neurons and Knowing: Constructivism, Coherence Psychology, and their neurodynamics substrates. Journl of Constructivist Psychology, 20, 3, 201-245.

Toomey, B., \& Ecker, B. (2009). Competing visions of the implications of neuroscience for psychotherapy. Journl of Constructivist Psychology, 22, 2, 95140.

Twenge, J. M., \& Campbell, W. K. (2003). "Isn't it fun to get the respect that we're going to deserve?" Narcissism, social rejection, and aggression. Personality and Social Psychology Bulletin, 29, 261-272.

Ugazio, V. (1998). Storie Permesse e Storie Proibite. Polarità Semantiche Familiari e Psicopatologia. Torino: Bollati Boringhieri.

Ugazio, V., Castiglioni, M. (1998). Socialization models and the construction of Self. Journal of Constructivist Psychology, 11, 3-29.

Ugazio, V., Fellin, L., Colciago, F., Pennacchio, R., \& Negri, A. (2008). 1 to 3: From the monad to the triad. A unitizing and coding system for the inference fields of causal explanations. TPM. Testing, Psychometrics, Methodology in Applied Psychology, 15, 171-192.

Westen, D. (1990). The relations among narcissism, egocentrism, selfconcept and self-esteem: Experimental, clinical and theoretical considerations. Psychoanalysis and Contemporary Thought, 13, 183-239.

Ricevuto: 20 gennaio 2009. Revisionato: 3 marzo 2009. Accettato: 25 settembre 2009 . 